\title{
Magnetoencephalography of Epilepsy with a Microfabricated Atomic Magnetrode
}

\author{
DiD Orang Alem, ${ }^{2}$ Alex M. Benison, ${ }^{1}$ Daniel S. Barth, ${ }^{1}$ John Kitching, ${ }^{2}$ and Svenja Knappe ${ }^{1,2}$ \\ ${ }^{1}$ Department of Psychology and Neuroscience, University of Colorado, Boulder, Colorado 80309, and ${ }^{2}$ Time and Frequency Division, National Institute of \\ Standards and Technology, Boulder, Colorado 80305
}

\begin{abstract}
Magnetoencephalography has long held the promise of providing a noninvasive tool for localizing epileptic seizures in humans because of its high spatial resolution compared with the scalp EEG. Yet, this promise has been elusive, not because of a lack of sensitivity or spatial resolution but because the large size and immobility of present cryogenic (superconducting) technology prevent long-term telemetry required to capture these very infrequent epileptiform events. To circumvent this limitation, we used Micro-Electro-Mechanical Systems technology to construct a noncryogenic (room temperature) microfabricated atomic magnetometer ("magnetrode") based on laser spectroscopy of rubidium vapor and similar in size and flexibility to scalp EEG electrodes. We tested the magnetrode by measuring the magnetic signature of epileptiform discharges in a rat model of epilepsy. We were able to measure neuronal currents of single epileptic discharges and more subtle spontaneous brain activity with a high signal-to-noise ratio approaching that of present superconducting sensors. These measurements are a promising step toward the goal of high-resolution noninvasive telemetry of epileptic events in humans with seizure disorders.
\end{abstract}

Key words: atomic; epilepsy; magnetomoeter; MEG; seizure

\section{Introduction}

Neuronal currents associated with brain activity produce minute extracranial magnetic fields detectable in the magnetoencephalogram (MEG), accompanied by electrical potentials recorded in the scalp EEG. Over the past 30 years, there has been intense interest in MEG because, although electrical potentials are attenuated and distorted by the heterogeneous resistive properties of the cranium that compromise the spatial resolution of EEG, the skull is transparent to magnetic fields (Cohen and Cuffin, 1983). MEG has long presented the promise of providing noninvasive images of brain activity, with a spatial and temporal resolution rivaling that obtained only through penetrating the skull by use of electrodes placed within the brain.

Nowhere is the potential of MEG more clinically relevant than in the surgical treatment of human epilepsy (Barth et al., 1982, 1984), where the origin of seizures cannot always be adequately determined from the scalp EEG, making invasive intracranial

Received Aug. 20, 2014; revised Sept. 10, 2014; accepted Sept. 17, 2014.

Author contributions: 0.A., A.M.B., D.S.B., J.K., and S.K. designed research; 0.A., A.M.B., D.S.B., and S.K. performed research; 0.A., A.M.B., D.S.B., and S.K. analyzed data; 0.A., A.M.B., D.S.B., J.K., and S.K. wrote the paper.

This work was supported by the U.S. Army Medical Research and Material Command Grant PR100040 and National Institute of Science and Technology. We thank John LeBlanc (Draper Laboratories) for the fabrication of sensor heads and Vishal Shah (QuSpin Inc.) for providing signal processing algorithms for the $\mu$ AMs. This work is a contribution of National Institute of Science and Technology, an agency of the U.S. Government, and is not subject to copyright.

Trade name is stated for technical clarity and does not imply endorsement by National Institute of Standards and Technology. Products from other manufacturers may perform as well or better.

The authors declare no competing financial interests.

Correspondence should be addressed to Dr. Daniel S. Barth, University of Colorado, Department of Psychology and Neuroscience, Boulder, C0 80309. E-mail: dbarth@psych.colorado.edu.

DOI:10.1523/JNEUROSCI.3495-14.2014

Copyright $\odot 2014$ the authors $\quad 0270-6474 / 14 / 3414324-04 \$ 15.00 / 0$ electrical recording (electrocorticography [ECoG]) necessary (Engel, 2013). Yet, the use of MEG to replace invasive recording of epilepsy has remained elusive, primarily because present cryogenic technology is not suited to long-term telemetry required to capture single rare events (seizures). What is needed to fully realize the clinical potential of MEG are sensors that may be operated at room temperature, can be directly and flexibly attached to the scalp (e.g., EEG electrodes) for long-term telemetry, are of sufficiently small size to permit high density placement, and most important, are capable of detecting single epileptic discharges with a sensitivity similar to invasive recording electrodes. Here, we report recording single epileptiform spikes and smaller-amplitude spontaneous brain activity, in an animal model where MEG could be directly compared with ECoG, using a microfabricated atomic magnetometer $(\mu \mathrm{AM})$ that meets these requirements.

\section{Materials and Methods}

$\mu A M$ principle of operation. Atomic magnetometers, also known as optically pumped magnetometers, use the influence of magnetic fields on alkali atoms in a vapor (see, e.g., Budker and Romalis, 2007). The vapor of atoms is spin-polarized by transferring the spin of resonant laser light to the atoms (Fig. 1A). Once the atoms (in our case rubidium) are polarized, a precession of the electron spin can be imparted by external magnetic fields (Fig. 1B). Reorientation of the spins changes the light transmitted through the atomic vapor. At zero magnetic field, the light transmission is maximum because most of the atoms are spin-polarized parallel to the transmitted light. In a nonzero transverse magnetic field, the atoms precess, and the static polarization, resulting from a balance between optical pumping and precession, will not be optimally aligned with the pump light, causing a decrease in the transmission intensity (Fig. $1 C)$. To determine the direction of the magnetic field, a small oscillating 
A
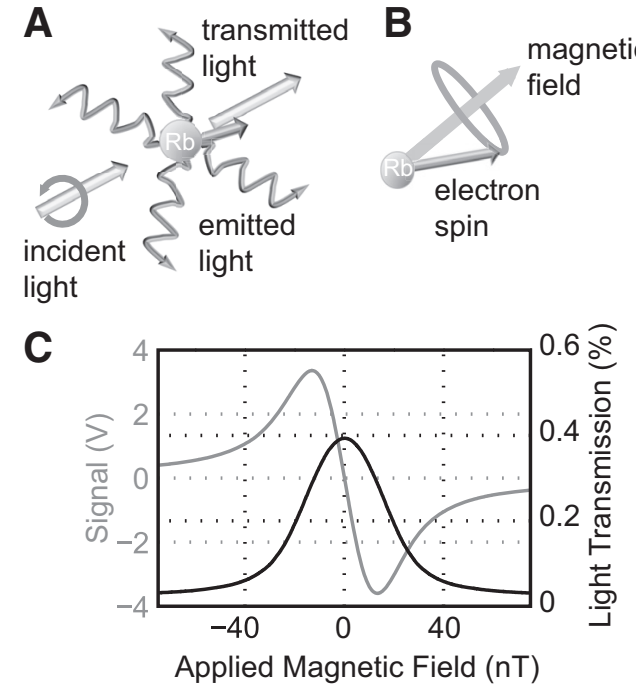

D

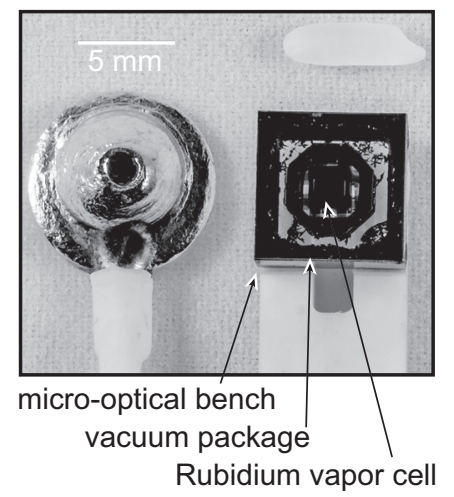

Figure 1. Theory and operation of the $\mu \mathrm{AM}$. $A$, Rubidium $\left({ }^{87} \mathrm{Rb}\right)$ atoms are spin-polarized by transferring the spin of resonant laser light. $\boldsymbol{B}$, Precession, or wobble, of electron spin is imparted by external magnetic fields, changing the light transmitted through the atomic vapor. C, Plot of light transmission with magnetic field strength (black; right) and the demodulated signal (gray; left). D, $\mu \mathrm{AM}$ (right) with photodiode removed to expose ${ }^{87} \mathrm{Rb}$ vapor cell inside of vacuum package. The $\mu \mathrm{AM}$ is shown compared with a standard scalp EEG electrode (left) and grain of rice (top).

magnetic field is applied perpendicular to the light beam, and the transmitted light is demodulated with a phase-sensitive detector.

$\mu A M$ construction. We built a $\mu$ AM (Schwindt et al., 2007) of comparable size to a standard scalp EEG electrode (Fig. 1D), using MicroElectro-Mechanical Systems technology. The use of fiber-optics and evacuated packaging (Mhaskar et al., 2012; Sander et al., 2012) enabled flexible positioning, low-power operation, and the ability to position the heated sensor within a few millimeters of living tissue. The sensor head contained a ${ }^{87} \mathrm{Rb}$ vapor cell within a vacuum package (Mescher et al., 2005) (Fig. 1D), keeping the outside of the sensor at room temperature while the cell was heated to $150^{\circ} \mathrm{C}$. The thin vacuum gap allowed a very high-signal-to-noise ratio because the atomic vapor could be positioned within millimeters of the scalp. The vacuum package was glued to a microfabricated optical bench (Fig. $1 D$, underside of $\mu \mathrm{AM}$ ) holding a ferrule with the optical fibers, along with a polarizer, beam splitter, mirror, and quarter-wave plate. The pump light emitted by one of the fibers was circularly polarized, transmitted through the rubidium cell, and collected on a photodiode (removed to expose vapor cell in Fig. 1D).

MEG measurement setup. A distributed-feedback laser was used to pump and probe the atoms in four sensors simultaneously. The light was coupled into a polarization-maintaining fiber and split into four arms, one for each sensor, by means of a fiber splitter with manually adjustable attenuation. The heating light was provided by a second distributedfeedback laser and split into four channels with a fiber splitter. The heat power for each sensor was adjusted individually with variable optical attenuators. The signal from the photodiode was amplified and pro- cessed with a digital signal processor-based lock-in amplifier. A low-pass filter of eighth order at $400 \mathrm{~Hz}$ and notch filters at $60 \mathrm{~Hz}$ and $180 \mathrm{~Hz}$ were applied digitally.

The sensor heads were mounted in a 3D-printed plastic holder such that all fibers were pointing in the vertical direction. The holder was mounted to a Delrin platform. The platform was slid into a Plexiglas cylinder used as a manifold to hold a set of magnetic field coils to control the magnetic-field environment. The coil set could create three orthogonal magnetic fields homogeneous to 1:100 over a volume of $5 \mathrm{~cm}$. In addition, a field gradient along the cylinder axis could be applied to the field along this axis. The coil manifold was housed in a three-layer magnetic shield made of $\mu$-metal. The rat was positioned on a slider bed with a nonmagnetic 3D-printed nose clamp and two ear bars. The bed could be shifted in two horizontal directions under the sensor array, and the sensors could be lowered to touch the head of the animal. Two sensors were mounted in the vertical direction with a separation of $1 \mathrm{~cm}$, and a third sensor was $10 \mathrm{~cm}$ away to monitor the background magnetic fields. All sensors measured the field component along the cylinder axis.

Animals and surgery. All procedures were conducted within the guidelines established by the University of Colorado Institutional Animal Care and Use Committee. Adult male Sprague Dawley rats $(n=3 ; 510-515 \mathrm{~g})$ were anesthetized to surgical levels using subcutaneous injections of ketamine $(71 \mathrm{mg} / \mathrm{kg}$ of body weight), xylazine $(14 \mathrm{mg} / \mathrm{kg})$, and acepromazine $(2.4 \mathrm{mg} / \mathrm{kg})$. Additional doses were administered every $60 \mathrm{~min}$ to maintain an anesthetic depth where the corneal and flexor withdrawal reflexes could barely be elicited. Animals were placed on a regulated heating pad to maintain normal body temperature $\left(37^{\circ} \mathrm{C}\right)$. Skin, fascia, and parts of the masseter muscle were removed to expose the dorsal and lateral aspects of the skull. Bilateral cranial windows $(2 \mathrm{~mm} \times 2 \mathrm{~mm})$ were surgically opened on the lateral side of the skull at the junction of the squamosal and parietal bones by use of a small Dremel tool with a $1 \mathrm{~mm}$ burr tip under a surgical microscope. The dura was reflected and the exposed cortex regularly doused with Ringer's solution containing the following (in mM): $135 \mathrm{NaCl}, 3 \mathrm{KCl}, 2 \mathrm{MgCl}_{2}$, and $2 \mathrm{CaCl}_{2}, \mathrm{pH} 7.4$, at $37^{\circ} \mathrm{C}$. At the conclusion of the experiment, the animals were killed by anesthetic overdose without ever regaining consciousness.

Electrical recording. Electrophysiology was performed using Ag wire ball-electrodes (ball diameter: $\sim 1 \mathrm{~mm}$; bare wire diameter: $250 \mu \mathrm{m}$; Teflon-coated diameter: $330 \mu \mathrm{m}$ ). The electrodes were positioned epipially through the cranial window in the squamosal bone. Recordings were referenced to a similar electrode secured over the occipital bone and were amplified ( $\times 500$; DAM80, World Precision Instruments), analog filtered (bandpass: 1-1000 Hz). The demodulated signals from the three $\mu \mathrm{AMs}$ and amplified field potentials from the ECoG electrodes were recorded with a 24-bit ADC system at a $10 \mathrm{kHz}$ sampling frequency. Both MEG and ECoG signals were subsequently digitally bandpass filtered (1-100 $\mathrm{Hz}$ ) using a zero-phase, second-order, Butterworth design. To evoke epileptiform spikes, the $\mathrm{GABA}_{\mathrm{A}}$ antagonist bicuculline methiodide (Tocris Bioscience; $100 \mu \mathrm{l}, 4 \mathrm{~mm}$, in $0.9 \%$ saline) was applied to the cortical surface subsequent to ketamine spike data collection. Epileptiform spiking from bicuculline methiodide application stabilized after $10 \mathrm{~min}$, at which point data were collected in 5 min blocks.

\section{Results}

The intrinsic sensitivity of the $\mu \mathrm{AM}$ was measured to be $<25$ $\mathrm{fT} / \mathrm{Hz}^{1 / 2}$ and averaged between 10 and $50 \mathrm{~Hz}$. The noise from the magnetic shield was estimated to be $\sim 12 \mathrm{fT} / \mathrm{Hz}^{1 / 2}$, and the total sensitivity inside the shield can was at $30 \mathrm{fT} / \mathrm{Hz}^{1 / 2}$ during the experiment. The intrinsic bandwidth of the sensor was $\sim 250 \mathrm{~Hz}$, rolling off similar to a first-order low-pass filter to a frequency set by the steep digital signal processor low-pass filter.

Figure 2 shows simultaneous recording from the $\mu \mathrm{AM}$ and from ECoG electrodes placed directly on the cortical surface of a rat. With the bottom of the $\mu \mathrm{AM}$ vacuum package pressed against the dorsal parietal skull, the center of the ${ }^{87} \mathrm{Rb}$ cell was located $\sim 3.7 \mathrm{~mm}$ dorsal to the left parietotemporal ridge in Experiment 1 (Fig. 2A), or left and right parietotemporal ridges in Experiment 2 (Fig. 2D). In Experiment 1, an ECoG electrode 



Figure 2. Simultaneous magnetic and electric recording of spontaneous spikes in the rat. $A$, Sagittal view of the rat skull (caudal is toward the right) showing positions of the ${ }^{87} \mathrm{Rb}$ cell for MEG, and parietal cranial window for ECOG, recording in Experiment 1. The circle with arrows indicates the direction of magnetic fields expected during the negative peak of spikes in the left hemisphere ECoG. At the level of the MEG sensor, the tangential component of the magnetic field is pointed caudally. $\boldsymbol{B}$, Top, Raw MEG (gray) and ECoG (black) of nonepileptiform ketamine spikes (*). Bottom, Five superimposed ketamine spikes (thin traces) and their average (thick traces). $\boldsymbol{C}$, Similar to $\boldsymbol{B}$ but showing an example of a single epileptiform spike after focal injection of bicuculline methiodide via the cranial window. Top, Bottom, Single unaveraged spikes. D, Dorsal view showing bilateral placement of magnetrodes over the parietotemporal ridges and bilateral parietal ECoG electrodes. $\boldsymbol{E}$, Averaged $(n=20)$ ketamine spikes using the left MEG response peak (arrow) to align the average. The MEG spike (gray) is lateralized to the left hemisphere and attenuated over the right. The averaged ECOG spike is confined to the left hemisphere. $\boldsymbol{F}$, Similar to $\boldsymbol{E}$ but using the right MEG response peak (arrow) to align averaging. Note a complete shift of the MEG and ECoG response to the right hemisphere. The magnetic field is reversed to a rostral direction, as expected for a right hemisphere equivalent dipole reflecting intradendritic current flowing away from the cortical surface toward the deeper cortical layers.

recorded data from the pial surface (dura reflected) of the left hemisphere through a cranial window in the temporal bone 10 $\mathrm{mm}$ ventral to the ${ }^{87} \mathrm{Rb}$ cell (Fig. $2 A$ ). In Experiment 2, bilateral ECoG monitoring was performed (Fig. 2D). The smallest signals recorded were spontaneous transient nonepileptiform discharges associated with ketamine anesthesia (Kayama and Iwama, 1972) ("ketamine spikes"; Fig. 2B, asterisk). The negative peaks of the ketamine spikes $(280 \pm 14 \mu \mathrm{V})$ were accompanied by similar spikes in the MEG $(1.3 \pm 0.18 \mathrm{pT})$ that could be visualized individually in the raw recording. In this figure, downward deflections of the MEG trace reflect a dorsal tangential magnetic field component pointed caudally. Thus, during the negative ECoG spike in the left hemisphere, the caudally pointed magnetic field was consistent with that generated by dipolar intradendritic currents perpendicular to and directed away from the cortical surface (Barth et al., 1986), producing clockwise magnetic fields (Fig. $2 A$, circle with arrows). The signal-to-noise ratios of ketamine spikes were $9.6 \pm 1.3$ and $76.2 \pm 10.6$ in the MEG and ECoG, respectively, when noise in the live animal (sampled between spikes) was used for comparison and $18.5 \pm 2.4$ and 197.2 \pm 10.6 , respectively, compared with noise after death. Examination of recordings before and after death indicated that higher noise in the live animal was due in part to spontaneous brain activity and not from other biological sources, such as respiration or cardiac artifact. Epileptiform spikes following cortical bicuculline me- thiodide injection at the cranial window were $\sim 3$ times larger than ketamine spikes in the ECoG (Fig. $2 C ; 888 \pm 32.7 \mu \mathrm{V}$; negative peak) with correspondingly large amplitude signals in the MEG $(2.8 \pm 0.18 \mathrm{pT})$. Signal-to-noise ratios in the MEG and ECoG of epileptiform spikes were also higher in the live (45.8 \pm 1.4 and $606 \pm 129.1$, respectively) and killed (105.8 \pm 20.7 and $855.6 \pm 145.8$, respectively) animals.

Experiment 2 demonstrated the spatial resolution of $\mu \mathrm{AM}$, with a capacity to discriminate small ketamine spikes originating in the opposite hemispheres. When we used the peaks of $\sim 20$ MEG spikes recorded above the left hemisphere (Fig. 2E, arrow) to time-align averages of the bilateral ECoG and MEG, the electrical and magnetic signals indicated a large source in the left hemisphere (Fig. 2E, bottom), an attenuated response in the right MEG, and no response in the right ECoG (Fig. 2E, top). The converse was true when ketamine spikes in the right MEG sensor were used for averaging (Fig. $2 F$, arrow), resulting in a large ECoG response in the right hemisphere; MEG responses also lateralized largely to the right hemisphere, and no corresponding signal in the left hemisphere ECoG (Fig. $2 F$ ).

\section{Discussion}

The spatial resolution and sensitivity of the $\mu \mathrm{AM}$ demonstrated here are comparable with the most sensitive animal measurements of single epileptiform spikes performed with cryogenic 
technology (Nowak et al., 1999; Zwiener et al., 2000). Because the $\mu \mathrm{AM}$ operates without a cryogenic Dewar and can therefore be directly attached to the scalp as a magnetrode, its sensitivity for stationary recording of human epileptic discharge should also be comparable with present cryogenic MEG systems used in magnetically shielded environments. Yet, the small size of the $\mu \mathrm{AM}$ also makes it ideally suited to portable systems that could be worn for long-term nonstationary telemetry of brain pathology, such as epileptic seizures in humans. Recent advances in scalar optically pumped sensors have shown sensitivities $<1 \mathrm{fT} / \mathrm{Hz}^{1 / 2}$ in centimeter-size cells (Sheng et al., 2013). The operation of scalar magnetometers improves the dynamic range and commonmode rejection of gradiometers. Microfabrication of such sensors can further reduce size and facilitate convenient recording in unshielded or minimally shielded environments required for practical human telemetry. Alternatively, further improvements in sensitivity of the current zero-field sensors to near the limit set by photon shot noise of $10 \mathrm{fT} / \mathrm{Hz}^{1 / 2}$ may also enable single-trial recordings of deeper brain structures in humans because the distance between sensor and scalp can be as small as $2.5 \mathrm{~mm}$. Although these technical improvements will facilitate the use of atomic MEG for human telemetry, the present animal recordings justify and motivate this work. The $\mu \mathrm{AM}$ represents an advance in neuro-magnetometry with the potential to realize the originally anticipated application of MEG to the noninvasive presurgical evaluation of human epilepsy by localizing the source of seizures.

\section{References}

Barth DS, Sutherling W, Engel J Jr, Beatty J (1982) Neuromagnetic localization of epileptiform spike activity in the human brain. Science 218:891894. CrossRef Medline
Barth DS, Sutherling W, Beatty J (1984) Fast and slow magnetic phenomena in focal epileptic seizures. Science 226:855-857. CrossRef Medline

Barth DS, Sutherling W, Beatty J (1986) Intracellular currents of interictal penicillin spikes: evidence from neuromagnetic mapping. Brain Res 368: 36-48. CrossRef Medline

Budker D, Romalis M (2007) Optical magnetometry. Nat Phys 3:227-234. CrossRef

Cohen D, Cuffin BN (1983) Demonstration of useful differences between the magnetoencephalogram and electroencephalogram. Electroencephalogr Clin Neurophysiol 56:38-51. CrossRef Medline

Engel J (2013) Seizures and epilepsy. New York: Oxford UP.

Kayama Y, Iwama K (1972) The EEG, evoked potentials, and single-unit activity during ketamine anesthesia in cats. Anesthesiology 36:316-328. CrossRef Medline

Mescher MJ, Lutwak R, Varghese M (2005) An ultra-low-power physics package for a chip-scale atomic clock. IEEE Solid-State Sensors Actuators 1:311-316.

Mhaskar R, Knappe S, Kitching J (2012) A low-power, high-sensitivity micromachined optical magnetometer. Appl Phys Lett 101:241105. CrossRef

Nowak H, Giessler F, Huonker R, Haueisen J, Röther J, Eiselt M (1999) A 16-channel SQUID-device for biomagnetic investigations of small objects. Med Eng Phys 21:563-568. CrossRef Medline

Sander TH, Preusser J, Mhaskar R, Kitching J, Trahms L, Knappe S (2012) Magnetoencephalography with a chip-scale atomic magnetometer. Biomed Opt Express 3:981-990. CrossRef Medline

Schwindt PDD, Lindseth B, Knappe S, Shah V, Kitching J, Liew LA (2007) Chip-scale atomic magnetometer with improved sensitivity by use of the Mx technique. Applied Phys Lett 90:081102-081103.

Sheng D, Li S, Dural N, Romalis MV (2013) Subfemtotesla scalar atomic magnetometry using multipass cells. Phys Rev Lett 110:160802. CrossRef Medline

Zwiener U, Eiselt M, Giessler F, Nowak H (2000) Relations between early prespike magnetic field changes, interictal discharges, and return to basal activity in the neocortex of rabbits. Neurosci Lett 289:103-106. CrossRef Medline 